\title{
Irrational Prescribing Practices: A Threat To Underdeveloped World
}

Afshan Mehreen*

Comsats institute of information technology Northern Punajb Rawalpindi, Pakistan.

*CorrespondingAuthor: Afshan Mehreen, Comsats institute of information technology Northern Punajb Rawalpindi, Pakistan. Email: afshan.mehreen@yahoo.com

Citation: Afshan Mehreen, (2017). Irrational Prescribing Practices: A Threat To Underdeveloped World. Int J Pharm Sci \& Scient Res.3:1,21-25.

Copyright: (C) Afshan Mehreen, This is an open-access article distributed under the terms of the Creative Commons Attribution License, which permits unrestricted use, distribution, and reproduction in any medium, provided the original author and source are credited.

Received February 9, 2017; Accepted February 21, 2017; Published February 28, 2017.

Abstract

A lot of data from the world is available to describe irrational use of drugs all around the world. Most of the studies have described the irrational prescribing practices by analyzing prescriptions in comparison with standard provided by World Health Organization WHO. Such irrational practices include not only over prescription, multidrug prescribing, use of unnecessary expensive drugs, overuse of antibiotics and injections but also the behaviors of prescribers in the particular region. To overcome such problems WHO has recommended different prescribing indicators, which could be the first step to be followed in order to promote rational use of drugs. To improve the financial and health status of the public, it is necessary to improve factors influencing drug use. Although more research work is still needed to assess socio cultural factors influencing the impact of drug use patterns, particularly from prescriber's perspective. A lot of data from the word is already available to assess such practices .there is a need to sort out the problems prevailing in developing countries regarding prescriptions. So it will be helpful in suggesting interventions to solve these problems.

Rationalization measures can help to lower costs, which in turn result in prices that are affordable for a larger proportion of the population

Keywords: Antioxidant, delayed bonding, shear bond strength, tooth bleaching

Introduction

General Prescribing practices and prescriber's behaviors in any country or region play a key role in deciding the health future of the community. For proper medication every member of the community is dependent upon physician of his/her locality. Physicians of any society have the freedom to take decisions regarding medication of the patients. As all the drugs are chemicals so should be taken with precautionary measures. Over and under use of these agents may lead to dependence and resistivity respectively. Rational use of drugs means that patients must receive medications that are accurate for their clinical needs in terms of doses that meet their individual demands, for sufficient period of time, and at the lowest cost. The demands for rational use will be meet if the process of prescribing is appropriately followed. This process includes steps in assessing patient's problems, deciding effective and safe treatments, selecting appropriate drugs, dosage, and duration. The rational use of drugs thus implies to take the medication at right time with right frequency having right dose and at the lowest cost. Therefore prescribing practices in context of irrational drug use should be monitored properly. For quality of a prescription to be assessed World Health Organization (WHO) has recommended a minimum of five core indicators overall. These parameters cover all the aspects to be monitored to promote rational use of drugs. These indicators include the number of drugs per prescription and percentage of generic drugs, antibiotics, injections and drugs from National Essential Drug List (NEDL) of the country. Irrational prescribing patterns also lead to increase the cost of therapy overall. Polypharmacy i.e. more number of drugs prescribed for the treatment which is not indicated is also one of the major problems facing worldwide on behalf of poor prescribing patterns and has now become a serious threat to the world, which not only is responsible for increase in cost but also a compromise to health. As the more the number of drugs prescribed the more is the non compliance of the patient to the therapy. Although it is not possible to prevent all prescription-related problems in older people, several studies have shown that it is possible to reduce the occurrence of prescriptionrelated problems by means of a medication review.

The types of prescribers also give an insight to their behavior as there is a difference in

approaches for the treatment of similar health problems. There are many evidences which showed that medical specialists have different approaches as compared to the general practitionerrs, where G.Ps are involved more in promoting irrational prescribing practices whenever compared with the specialists.

The prescribing indicators as provided by WHO are taken 
as standard because keeping all of them in view one is able to describe what intervention should be applied to correct the problem related to prescription. If any one of these core indicators is not in accordance with the standard, it will lead to poor health. The number of drugs per prescription gives an idea of poly pharmacy and must be monitored in order to promote rational use of drugs as high number of drugs is not only responsible for high cost but also compromised health status. The average number of drug items prescribed per patient is considerably higher in many countries, particularly in urban areas as people living in these areas get treatments from general practitioners because of low cost and easy access to them.

\section{Methods}

The term irrational in this overview denotes wrong prescribing and misuse of therapeutic agents. The studies evaluating prescription data in comparison with the standard core prescribing indicators by WHO were identified and included. Studies that contained quantitative data on these prescribing indicators were included. The studies not only from the underdeveloped world but also from the world were included to describe the health status influenced by the inappropriate use of drugs. The issues addressed in this overview are the existence of irrational drug use by the prescribers in terms of violation of standard core indicators and reasons for their existence. This overview has not only mentioned the prescribing practices but also the drug use problems associated with irrational use and the possible consequences related.

\section{Drug Resistance}

The over/under use of some antibiotics has lead to existence of resistant pathogens. The most common example of drug resistance facing worldwide is the antibiotic resistance to different strains of microorganisms. The number of antibiotics prescribed is helpful in defining the reason for existence of antibiotic resistance, as unnecessary use of antibiotics without having proper indication is the leading cause. In underdeveloped world the ratio of unnecessarily prescribed antibiotics is relatively high because the rate of infectious diseases is high1.

Resistance to first-line, inexpensive, broad-spectrum antibiotic is increasing. The selective pressure exerted by over prescription of drugs has greatly increased the rate of bacterial resistance2. Unskilled practitioners play major role in this context.

Major contribution to drug resistance is due to unnecessary use of antibiotics in diarrhea. ORS is mostly received by the children taken to public health centers as compared to general practitioners3. The use of ORS is mostly dependent on differences in behaviors of providers. Prescribers more often suggest ORS for the children who suffer dehydration.

Another major reason for antibiotic prescription is upper respiratory tract infections 4 . Overuse of antibiotics persists across physician specialties which are involved in improving clinical practice. Irrational prescribing of antibiotics for acute cough is responsible for patient's expectations to be treated by same agents when experience same symptoms every time. The no of drugs per prescription is greater than standard and the no of antibiotics per prescription is also very high5,6. In developing countries rate of morbidity and mortality due to bacterial infections is relatively high7,8. This is because most of the population in the developing countries has inadequate medical care, poor sanitation and nutrition due to poverty. The prevalence of these infections has lead to the over prescription of antibiotics in these regions. As for example the main causes of morbidity in Nepal are gastrointestinal and respiratory infections, enteric fever, tuberculosis and malnutrition9.

Due to poor economic status the people living in developing countries most conveniently visit general practitioners rather than specialists. These G.Ps are involved in irrational prescribing of antibiotics. G.Ps prescribe antibiotics for acute watery diarrhea and acute respiratory tract infections, 10, 11, 12 .

One of the studies also showed that most commonly prescribed therapeutic agents by prescribers are systemic antibacterials and pantoprazole13.

\section{Polypharmacy}

Polypharmacy is defined as multiple drugs prescribed by the physician or administration of more medicines than are clinically indicated, leading to unnecessary use of drugs. Major risk factors contributing to polypharmacy are age, race/ethnicity, sex, educational achievement level, health status, and number of concomitant diseases. Polypharmacy may lead to serious problems such as drug-drug interactions, duplication of therapy, non adherence, and adverse effects 14 . The minimum number of drugs describing polypharmacy ranges from 5 to 10 . The diabetes, major cause of polypharmacy include multiple prescribers, aging population, complex drug therapies, psychosocial contributions, adverse drug reactions that may be interpreted as new medical conditions. Over prescription is not only harmful for health but is also responsible for increased cost of therapy 15 . The greater the number of drugs prescribed the greater is the number of drugs dispensed incorrectly16. Polypharmacy is more commonly experienced by patients of 75 years of age or older with multiple comorbidities 17. Thus elderly persons are exposed more to polypharmacy 18,19 . The average number of drugs per prescription is high as compared to the standard. The proposed no of therapeutic agents per prescription by WHO is less than 2 but actual no of these agents prescribed is greater than this value, 20, 21,22,23.

The no of drugs prescribed irrationally by G.Ps is more as compared to public health sector, 24,25 ,

\section{Generic Prescription}

No of drugs prescribed by their generic names is also included by WHO in prescribing

indicator's list. The name given to the drugs by manufacturers is known as brand name. There exists a price difference between different brands of the same drugs. If drugs in the prescription are in generic then it is easy for the patient to choose the cost effective medicine according to their economic status. Generic prescription not only helps in minimizing the cost but also decreases the chances of over prescription. There are evidences of prescribing same 
agents with different brands. Most of the studies clearly showed that prescribers prefer brand names over generic. The percentage of medicines prescribed by their generic names is really low violating WHO's core indicators28, 29, 30, 31. Combination preparations containing 'hidden' classes of drug are prescribed commonly 32.

\section{Overprescription of Drugs}

Over prescription of drugs reflects the unnecessary prescription of the therapeutic agents. Physicians prescribe injectables, multivitamins and pain killers unnecessarily. This is mainly due to presence of gap between actual practice of prescribers and the recommended prescribing practice.

The drugs prescribed the most all over the world are NSAIDs. These agents are considered the least harmful among all the therapeutic categories but they are actually responsible for many gastrointestinal events 33,34 . Overuse of anti-infectives is also widely associated with irrational prescribing 35 .

In a literature review published in 2003 it is mentioned that the estimated no of injections administered annually in developing and transitional countries is 16 billion, $95 \%$ of which are used for curative purposes 36 . The misuse of injectables by the prescribers is often due to the demand by the patients 37 . Thus over use of injectables is also common by prescribers 38 .

\section{Limitations of The Overview}

It was difficult to find references for unpublished researches. The articles included in this

overview show only one side of the picture from prescribers' perspective whereas irrational use of drugs could be due to irrational dispensing as well as self medication. Thus there is a need to review all the articles covering all the aspects of irrational use of drugs. It would not have been possible to review all the available data due to lack of sources available and time. Underdeveloped world is at risk of losing healthy population on behalf of irrational prescribing patterns. The prescription data of the countries of third world clearly shows that consultants in these areas are violating rational practice $39,40,41,42$.

\section{Discussion}

This review indicates the existence of irrational prescribing patterns in the world. The situation is worse in developing countries including Pakistan. The results of articles cited clearly indicate the need for implementing proper intervention to enhance the rational use of drugs. First step in promoting rational drug use is to identify the problem and then to decide what preventive measures should be taken to solve these problems. Prescriptions could be analyzed and feedback could be provided to the prescribers. Consequences could be discussed with them in order to convince them to change their behavior for proper use of medication. In countries like Pakistan there is a need to change the approach towards therapy of the practitioners as they are contributing to increase the morbidity and mortality. Overuse of injections have lead the population to be at risk of acquiring blood-borne infections so measures must be taken to reduce the risk factors associated with unsafe usage of injections. There is trend of prescribing antibiotics irrespective of the need as in case of diarrhea. This is the factor responsible for drug resistance and prescribers must be convinced to change their behavior in order to reduce risk factors associated with resistance.

A prescription also reflects the prescribers' attitude to the disease and the nature of the health

care delivery system. As prescription is the form in which prescribers suggest the intervention for a particular problem. In developing countries where people are less educated and are dependent on physicians for their treatment. Prescription for them is the way to get rid of the disease by having medication according to their prescriber's suggestion. So first step to be taken for promotion of rational use of drugs is to motivate prescribers and ultimately it would help to improve the health status of these countries. Different studies also revealed that the violation of rational practices is much higher in Pakistan as compared to other regional countries that do have the same socio-economic pattern of population and health status of population.

\section{Recommendations}

The challenge is to change the approach of prescribers towards any particular disease state. First step to be taken in this context is to identify the reason for prevalence of irrational prescribing among doctors in these regions. This review clearly indicates that irrational prescribing practices are significantly present in developing countries specially Pakistan. In order to improve the health status there is a need to improve the overall health circle involved in disease identification, suggesting better intervention and making it certain to for the intervention to be followed according to the standard guidelines. This could be the way by which doctors can maintain the quality of their response to their patients.

\section{References}

1. Iruka N.O, Adebayo L and Robert E. Socioeconomic and behavioral factors leading to acquired bacterial resistance to antibiotics in developing countries. Emerging infectious diseases, january.february 1999 (5) 1.

2. Emmanuelle $\mathrm{C}$ and Patrice C. Antibiotic resistance in listeriaspp. Antimicrob. agents chemother. september 1999 (43) 9, 2103-2108.

3. Sivagnanam G, and Mohanasundaram J. A survey on current attitude of practicing physicians upon usage of antimicrobial agents in southern part of India. Medgenmed. 2004; (6) 2: 1 published online 2004 may 12 .

4. Anuja A.P, Subhash B.T and Prakash R.B. Prescription Analysis of Pediatric Outpatient Practice in Nagpur City. Indian J Community Med. 2010 January; 35(1): 70-73.

5. Kathryn M. Y. Provider bias in the treatment of diarrhea among boys and girls attending public facilities in Minia, Egypt. Social science \& medicine (56) 2003 753-768.

6. Charles P L, Unnati R S, Rafiqul I and Nikhil R. Childhood diarrhea management practices in Bangladesh: private sector dominance and continued inequities in care. Int. j. epidemiol. 
December 2006 (35) 6: 1430-1439. first published online: September 22, 2006

7. Nizami SQ, Khan JA, Bhutta ZA. Differences in self-reported and observed prescribing practice of general practitioners and paediatricians for acute watery diarrhoea in children of Karachi, Pakistan. J diarrhoeal dis res. 1995 mar; (13) 1:29-32.

8. Nizami SQ, Khan JA, Bhutta ZA. Drug prescribing practices of general practitioners and paediatricians for childhood diarrhoea in Karachi, Pakistan. J diarrheal dis res. 1995 mar; (13) 1:29-32.

9. Olivier T.R and Marisa E.D. Antibiotics for upper respiratory tract infections in

ambulatory practice in the United States, 1997-1999: does physician specialty matter. 196 JABFP may-June 2004 (17) 3.

10. Inayat H.T, Trudy H, Barbara M and Paul G. Private practitioners in the slums of Karachi: what quality of care do they offer. Social science \& medicine. (46) 11, 1 January 1998, 1441-1449.

11. Awad A.I, Ball D, Eltayeb I.B. Improving rational drug use in Africa: the example of Sudan. East mediterr health j. 2007 sep-oct; (13) 5:1202-11.

12. Ravi $\mathrm{S}$ and Praveen P. Prescribing patterns in medical outpatients. clinmed/2002050004 v1 (may 16, 2002)

13. Abdul H, Kiani A.G, Khalid B, Shah Z, Mirza Z. Prescription and dispensing practices in public sector health facilities in Pakistan: survey report. JPMA 54:187; 2004.

14. Najmi MH, Hafiz RA, Khan I, Fazli FR. Prescribing practices: an overview of three teaching hospitals in Pakistan. J pak med assoc. 1998 mar; (48) 3:73-7.

15. Anand H, Jinta J, Gurumurthy P, Madhan R, Shoba G. Prescribing patterns and predictors of high-level polypharmacy in the elderly population: a prospective surveillance study from two teaching hospitals in India. The American $\mathrm{j}$ of geriatric pharmacother (8) 3, June 2010, 271-280.

16. Treharne G.J, Douglas KM..J , Iwaszko J ,Panoulas V.F, Hale D, Mitton D, Piper H, Kitas D. Polypharmacy among people with rheumatoid arthritis: the role of age, disease duration and comorbidity. Musculoskeletal care December 2007, (5) 4, 175 190.

17. Stewart RB and Cooper JW. Polypharmacy in the aged. Practical solutions. Drugs \& aging 1994, (6) 4:449-61.

18. Wilma D. Analysis of polypharmacy in older patients in primary care using a multidisciplinary expert panel. BR J gen pract. 2006 july 1 ; (528) 56: 504-510.

19. Veehof, Stewart R.E, Haaijer-ruskamp F.M and Meyboom-de jong B. The development of polypharmacy. a longitudinal study. Family practice 2000 (17) 3: 261-267.

20. Jennifer Y.F, Wilson Y.S.L and Sophie C. Effectiveness of telephone counselling by a pharmacist in reducing mortality in patients receiving polypharmacy: randomised controlled trial. BMJ 2006; (333) (published 7 September 2006).

21. Kathleen A.H, Bharat R.G, Barnaby C.R. The effects of different kinds of user fees on prescribing quality in rural Nepal. J of clinical epidemiol. October 2001 (54) 10, 1065-1071.

22. Roger P. A. Polypharmacy as a risk factor in the treatment of type 2 diabetes. Pharmacy update. Diabetes Spectrum January 200619 (1).

23. Das N, Khan AN, Badini ZA, Baloch H, Parkash J. Prescribing practices of consultants at Karachi, Pakistan. J pak med assoc. $2001 \mathrm{Feb}$; (51) 2:74-7.

24. Hiroto I, Asuka K and Teruhiko H. Polypharmacy and excessive dosing: psychiatrists' perceptions of antipsychotic drug prescription. BJP 2005, (187):243-247

25. Arshad A, Zafar F, Agha A, Tanweer H, Henna Q. Determinants of therapeutic injection overuse among communities in Sindh, sauerborn. Prescription practices of public and private health care providers in Attock Pakistan. Vikram P, r vaidya, d naik, $\mathrm{p}$ borker. Irrational drug use in india: a prescription survey from Goa. JPGM, 2005 (51) 1: 9-12.

26. Kshirsagar M.J, Langade D, Patil S, and Patki P.S. Prescribing patterns among medical practitioners in pune, India. Bull world health organ. 1998; (76) 3: 271-275

27. Melinda P, Talgat N, Grace H, Farruh Y, Richard L. Prescribing practices of rural primary health care physicians in Uzbekistan. Tropical med \& international health. February 2003 (8) 2: 182190.

28. Roy C, Parameswar R, Gupta U, Sharma S, Tekur U and Bapna J.S. Quality medicines for the poor: experience of the Delhi programme on rational use of drugs. Health policy and planning; (20) 2: 124-136.

29. Nwolisa, Erinaugha and Ofoleta, prescribing practices of doctors attending to under fives in a children's outpatient clinic in owerri, Nigeria. Oxford j med, journal of tropical pediatrics (52) $3,197-200$.

30. Suh D.C0 , Hunsche E, Shin H.C and Mavros P. Co-prescribing of proton pump inhibitors among chronic users of NSAIDs in the UK. Rheumatology 2008(47) :458-463.

31. Robyn T, Laeora B, Dale D, David G, Roland G, Allen H, Lisa I, Peter M and Linda S. Unnecessary prescribing of NSAIDs and the management of NSAID-related gastropathy in medical practice. Ann intern med. 1997 (127):429-438.

32. Hafeez , Kiani A.G and Muhammad. Prescription and dispensing practices in public sector health facilities in Pakistan: survey report. JPMA April, 2004.

33. Tazeen H. J, Saleem J, Fahim H.J, Mohammad I, Raza O, Sarwar O, Andrew SL, Nish C. General practitioners' approach to hypertension in urban Pakistan disturbing trends in practice. 
AHAJ. Circulation. 2005; (111): 1278-1283.

34. Rennie M.D. Role of health-seeking behaviour in child mortality in the slums of karachi, Pakistan. J of biosocial science 2003 (35) : 131-144

35. Benjamin S.C, Uzochukwu, Obinna-e-onwujekwe and Cyril O.A. Effect of the bamakoinitiative drug revolving fund on availability and rational use of essential drugs in primary health care facilities in south-east Nigeria. Health policy plan. (2002) (17) $4: 378-383$

36. Trisha G. Drug prescription and self-medication in India: an exploratory survey, social science \& medicine (25) 3, 1987, $307-$ 318.

37. Kamran S, James N and Mike R. Getting evidence into practice: what works in developing countries. Int $\mathrm{j}$ qual health care October 2005 (17) 5: 447-454. first published online: may 4, 2005

38. Gaash B. Irrational use of antibiotics. Indian $\mathrm{j}$ for the practising doctor, (5) 1 (2008-03 - 2008-04) 12countries.

39. Ramesh B. Characteristics of private medical practice in India: a provider's perspective. Health policy and planning; (14) 1: 2637. 